\title{
Simultaneous pure laparoscopic resection of primary colorectal cancer and synchronous liver metastases: a single institution experience with propensity score matching analysis
}

\author{
Arpad Ivanecz',3, Bojan Krebs ${ }^{13}$, Andraz Stozer², Tomaz Jagric ${ }^{1}$, Irena Plahuta1, \\ Stojan Potrc ${ }^{1,3}$
}

\author{
${ }^{1}$ Department of Abdominal and General Surgery, University Medical Center Maribor, Maribor, Slovenia \\ 2 Institute of Physiology, Faculty of Medicine, University of Maribor, Maribor, Slovenia \\ ${ }^{3}$ Department of Surgery, Faculty of Medicine, University of Maribor, Maribor, Slovenia \\ Radiol Oncol 2018; 52(1): 42-53.
}

Received 8 September 2017

Accepted 8 October 2017

Correspondence to: Dr. Arpad Ivanecz, Department of Abdominal and General Surgery, University Medical Center Maribor, Ljubljanska ulica 5, 2000 Maribor, Slovenia. E-mail: arpad.ivanecz@ukc-mb.si

Disclosure: No potential conflicts of interest were disclosed.

Background. The aim of the study was to compare the outcome of pure laparoscopic and open simultaneous resection of both the primary colorectal cancer and synchronous colorectal liver metastases (SCLM).

Patients and methods. From 2000 to 2016 all patients treated by simultaneous resection were assessed for entry in this single center, clinically nonrandomized trial. A propensity score matching was used to compare the laparoscopic group (LAP) to open surgery group (OPEN). Primary endpoints were perioperative and oncologic outcomes. Secondary endpoints were overall survival (OS) and disease-free survival (DFS).

Results. Of the 82 patients identified who underwent simultaneous liver resection for SCLM, 10 patients underwent LAP. All these consecutive patients from LAP were matched to 10 comparable OPEN. LAP reduced the length of hospital stay $(P=0.044)$ and solid food oral intake was faster $(P=0.006)$ in this group. No patient undergoing the laparoscopic procedure experienced conversion to the open technique. No difference was observed in operative time, blood loss, transfusion rate, narcotics requirement, clinical risk score, resection margin, RO resections rate, morbidity, mortality and incisional hernias rate. The two groups did not differ significantly in terms of the 3-year OS rate (90 vs. $75 \% ; P=0.842$ ) and DFS rate (60 vs. $57 \% ; P=0.724)$.

Conclusions. LAP reduced the length of hospital stay and offers faster solid food oral intake. Comparable oncologic and survival outcomes can be achieved. LAP is beneficial for well selected patients in high volume centers with appropriate expertise.

Key words: colorectal cancer; synchronous liver metastases; laparoscopy; liver resection; colorectal resection

\section{Introduction}

Colorectal cancer is one of the most common causes of cancer related death in the Western world. ${ }^{1}$ At the time of diagnosis of the primary tumor, up to $25 \%$ of patients present with synchronous colorectal liver metastases (SCLM). ${ }^{2}$ Complete surgical re- section of both primary tumor and SCLM remains the only treatment option providing long-term survival and chance for cure. ${ }^{3}$

Different oncological strategies have been described including traditional two-stage colon first approach, ${ }^{4}$ liver first procedure ${ }^{5}$ or a one-step surgical resection of both the primary tumor and 
SCLM. ${ }^{6}$ Several reports have shown the benefit of a simultaneous open resection of primary tumor and SCLM compared with a staged approach. ${ }^{7-8}$

Minimally invasive colorectal and liver surgery are both accepted worldwide. ${ }^{9-11}$ Technical refinements and the development of advanced laparoscopic techniques has made simultaneous resection an attractive option. However, despite the increasing use of laparoscopy in colorectal and liver resections, simultaneous pure laparoscopic resection (SPLR) of the primary colorectal cancer and SCLM is still rarely performed.

Sporadic case reports and single institution series have shown the feasibility and safety of simultaneous laparoscopic resection of both primary CRC and SCLM. ${ }^{12-28}$ Recently, two large international multicenter retrospective studies, which were published from the same group of surgeons, confirmed that in selected patient's laparoscopic surgery allowed similar outcomes compared with the traditional open approach. ${ }^{29-30}$

The aim of this study was to compare the surgical and oncological outcomes of patients undergoing simultaneous resection of primary colorectal cancer and SCLM by laparoscopic or open surgery using propensity score matching.

\section{Patients and methods}

\section{Patient selection and study design}

All patients with SCLM were discussed by the multidisciplinary team. Treatment decisions were based on location and complexity of resection of the primary tumor, extent of liver resection, liver function and physical condition of the patients. The policy of the institution is not to combine a simultaneous major liver resection ( $\geq 3$ segments) with complex colorectal procedure (e.g. total colectomy, low anterior resection). The most ideal candidates for combined laparoscopic liver resection were patients with solitary, peripherally located metastasis in anterolateral segments.

Study subjects were identified from a prospectively maintained database of patients who underwent liver resections for CLM from January 2000 to December 2016 at the Department of Abdominal and General Surgery, University Medical Centre Maribor. This institution is a tertiary referral center with more than 15 years' experience in laparoscopic colorectal and with 8 years' experience in laparoscopic liver surgery. SCLM were defined as those identified at the time of diagnosis of the primary colorectal cancer. All patients gave their informed consent. Ethical approval for this study was obtained from the local institutional review board. Patient records were anonymized and deidentified prior to analysis.

All patients in the database who submitted to simultaneous laparoscopic resection of both the primary tumor and SCLM were selected and included in the study. Those laparoscopic patients were compared to patients that underwent open simultaneous liver and colorectal resection for stage IV colorectal cancer. A propensity score matching was applied to identify the most comparable patients from the open surgery group. Primary endpoints of the study were perioperative and oncologic outcomes. Secondary endpoints of the study were overall survival (OS) and disease-free survival (DFS).

\section{Neoadjuvant and adjuvant therapy}

Patients with rectal advanced local disease ( $\geq$ T3 and/or $\geq$ N1) and SCLM received short-course preoperative radiotherapy ( $5 \times 5 \mathrm{~Gy})$ and neoadjuvant systemic chemotherapy (3-6 courses) as a standard treatment protocol. However, neoadjuvant systemic therapy was not administered routinely for patients with colon cancer and SCLM. Adjuvant systemic therapy was administered at the discretion of the medical oncologist.

In general, chemotherapy included fluoropyrimidine-based therapies in combination with oxaliplatin or irinotecan. The different chemotherapy protocols included the FOLFOX (oxaliplatin, fluorouracil, and leucovorin), XELOX (capecitabin and oxaliplatin), XELIRI (capecitabin and irinotecan) and FOLFIRI (irinotecan, fluorouracil, and leucovorin) protocol, respectively. From year 2006, antiangiogenic agents (bevacizumab or cetuximab) were also added to these protocols.

\section{Simultaneous liver and colorectal surgery: surgical technique}

Surgical procedures were performed by one dedicated team and the liver resection was completed first. All liver resections (laparoscopic and open) were executed by at least one experienced senior HPB surgeon (SP or AI). A liver parenchymal sparing approach was applied in both groups. All laparoscopic colorectal resections were performed by one experienced senior colorectal surgeon (BK).

\section{Laparoscopic procedure}

Only pure laparoscopic liver and colorectal resections were performed. Generally, patients were 
placed in the supine position, except for resection of posterosuperior segments of the liver when the left lateral decubitus position was used. For rectal resections a split leg position was used. Four 12 $\mathrm{mm}$ ports were always used and additional $5 \mathrm{~mm}$ ports were placed as necessary. Laparoscopic ultrasonography of the liver was routinely performed to complete staging, locate the metastases, and accurately assess its margins and also to locate any adjacent biliary/vascular structures. It was also used to mark the plane of transection. Carbon dioxide pressure for pneumoperitoneum was kept at 12-14 mmHg during hepatic parenchymal transection. Pressures higher than this are generally avoided to reduce the risk of gas embolism but a slight rise during bleeding can aid hemostasis. The surface of the hepatic parenchyma was precoagulated with a $1-\mathrm{cm}$ surgical margin using monopolar coagulation. Liver transection was performed under low ( $<5 \mathrm{mmHg}$ ) central venous pressure. Pringle's maneuver was used selectively. Hepatic transections were performed using different high energy devices according to surgeon's preference (bipolar coagulation, thermofusion, ultrasound section or ultrasonic surgical aspirator). Larger structures were controlled with endoclips and endoscopic linear stapler devices were used selectively for division of portal pedicles and hepatic veins or their branches. The resected liver specimen was placed in a plastic bag and extracted after finishing the colorectal procedure. It was retrieved either through a suprapubic, a prolonged $12 \mathrm{~mm}$ port site or a short midline incision.

Laparoscopic colorectal surgery was performed according to standard oncologic procedures.

\section{Open resection}

A long midline incision was routinely performed which was extended to right subcostal incision whenever needed to adequately expose the posterosuperior segments of the liver. Intraoperative ultrasonography was routinely performed to guide resection. Hepatic transection was performed under low central venous pressure and intermittent pedicle clamping was used only in case of bleeding. Abdominal drains for liver resection were selectively used.

Colorectal resection routinely involved proximal ligation of vessels (the inferior mesenteric artery for the left colon and rectum, and the ileocolic artery for the right colon) and partial or total mesorectal excision depending on the location of the rectal cancer. After right colon resection a reconstruction was a hand-sewn ileocolic anastomosis.
For cancer located in the left colon and the rectum, reconstruction was a stapled colorectal anastomosis. A protective ileostomy was always performed for a low anastomosis. Abdominal drains were always used for rectal resections.

\section{Follow up}

Patients were followed at outpatient clinics at periodic intervals. Follow-up included physical examination, biochemical carcinoembryogenic antigen (CEA) test, thoracic X-ray or computed tomography (CT), and liver ultrasound, CT or magnetic resonance imaging (MRI) evaluation every 3 months for the first 2 years, and every 6 months thereafter.

Metastatic recurrence was diagnosed by CEA rise and $\mathrm{CT}$ or MRI. Positron emission tomography (PET) - CT scans were carried out in selected patients. In any uncertainty, histology was required.

Follow-up data were obtained from outpatient follow-up and from the National Cancer Register of Slovenia. Patient follow-up included details of dates of disease recurrence and death, site of recurrence, further therapy (e.g. systemic therapy, surgery), and cause of death. Recurrences were classified as hepatic, extrahepatic or combined. Recurrent disease was treated according to standard clinical practice and included surgery and/or chemotherapy whenever possible. The principles behind the selection criteria for resecting recurrent CRLM were the same as those for the initial hepatectomy.

Follow up was fully completed for all patients included in this study. All patients were followed up until their death or until March 2017.

\section{Primary endpoints: variables selected for analysis of the perioperative and oncologic outcomes}

Several routinely available clinical variables were analyzed and were divided in four groups:

- Preoperative clinical variables included baseline characteristics of patients, primary colorectal tumor and synchronous liver metastases. Herein any neoadjuvant therapy was included in the analysis;

- Intraoperative - simultaneous liver and colorectal surgery related variables;

- Postoperative oncological results after patohistology and clinical risk score (CRS);

- Postoperative outcome: patient's recovery, morbidity, mortality and incisional hernia rate.

Performance status was defined according to the American Society of Anesthesiologists (ASA). 
Location of SCLM were defined as anterolateral (segments 2, 3, 4b, 5, 6) or posterosuperior (segments $1,4 a, 7,8)$. The liver anatomy and resection terminology were based on the Brisbane classification. ${ }^{31}$ Hepatic resections were considered major when at least three adjacent segments were removed and defined as minor if $<3$ liver segments were resected. Conversion to an open operation was defined as an abdominal incision larger than that needed for specimen retrieval. CRS (from 0 to 5 ) as defined by Fong was applied. ${ }^{32}$ Briefly, patients with lower CRS tends to have a better prognosis. The histological surgical margin was defined as microscopically positive $(<1 \mathrm{~mm}, \mathrm{R} 1)$ or negative (R0). $\mathrm{R} 0$ resection was defined as complete removal of the tumors with a clear microscopic margin and without residual tumors. Complication was defined as any deviation from the normal course of recovery with the need for pharmacological, surgical, radiological, or endoscopic intervention. Postoperative morbidity was classified according to the Clavien-Dindo classification. ${ }^{33}$ Morbidity and mortality were defined as complications or death occurring within 90 days of surgery, or at any time during the postoperative hospital stay.

\section{Secondary endpoint: survival analysis}

Overall survival (OS) was defined as the interval between the date of first therapy (the date of first cycle of neoadjuvant therapy; if it was not applied then the date of simultaneous resection) and the date of death or the date of the last follow-up in surviving patients. Disease-free survival (DFS) was calculated from the date of first therapy (neoadjuvant therapy or simultaneous resection) to the date of intra- and/or extrahepatic recurrence or the date of the last follow-up in patients with no recurrence.

\section{Statistical analysis}

SigmaPlot 11.0 for Windows (Systat Software, Inc, CA) was used for statistical computations. Because of the inherent bias between patients undergoing laparoscopic and open surgery in terms of preoperative clinical characteristics, a 1:1 propensity score-matched analysis have been used to adjust for these differences. ${ }^{34}$ In this setting, propensity score adjustment was performed on the factors known to influence the choice of the approach. These factors included ASA score, primary tumor location (colon or rectal cancer), size, location, distribution and number of liver metastases and extent of liver surgery.

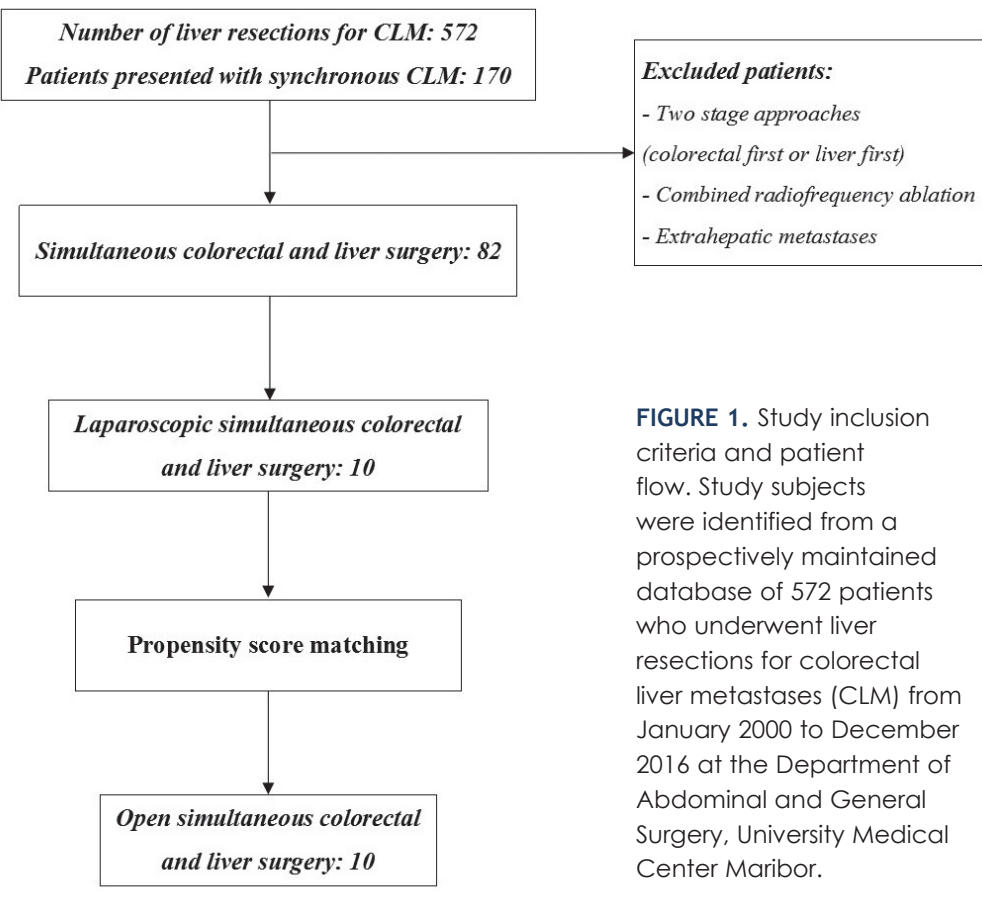

Differences in the frequency distributions of preoperative, intraoperative and postoperative clinical variables in relation to open and laparoscopic surgery were tested using the chi-squared test for categorical variables (Pearson's or Fisher's exact test when appropriate, two-tailed in all instances). Continuous variables were analyzed using Student's t-test for independent samples or the Mann-Whitney $U$ test if the criteria for a parametric testing were not met. The effects of open and laparoscopic surgery on overall and disease-free survival probabilities were estimated by using the Kaplan-Meier survival analysis and compared by using the log-rank test. A difference with a P value of $<0.05$ was considered statistically significant.

\section{Results}

\section{Study population and preoperative characteristics}

Of the 572 patients identified who underwent liver resections for CLM during the study period, simultaneous resection of both the primary tumor and SCLM were performed in 82 patients. From these, 10 patients were submitted to simultaneous pure laparoscopic procedure. After propensity score matching, all these 10 patients operated laparoscopically (LAP) were compared with 10 patients 
TABLE 1. Preoperative clinical characteristics of patients, primary colorectal tumor and synchronous colorectal liver metastases

\begin{tabular}{|c|c|c|c|}
\hline Variable & $\begin{array}{l}\text { Simultaneous Open } \\
\qquad(n=10)\end{array}$ & $\begin{array}{c}\text { Simultaneous } \\
\text { Laparoscopy } \\
(n=10)\end{array}$ & $\mathbf{P}$ \\
\hline \multicolumn{4}{|l|}{ Patients } \\
\hline Gender (male/female) & $6 / 4$ & $6 / 4$ & 1.00 \\
\hline Age $[$ mean $\pm S D$ (years)] & $65.4 \pm 8.1$ & $62.2 \pm 7.9$ & 0.39 \\
\hline BMI [median (IQR) (kg/m2)] & $24.0(23.1-25.5)$ & $26.9(23.6-32.1)$ & 0.34 \\
\hline ASA (I /II/III) & $4 / 3 / 3$ & $5 / 3 / 2$ & 0.86 \\
\hline Preoperative chemotherapy $(y / n)$ & $3 / 7$ & $7 / 3$ & 0.18 \\
\hline Preoperative radiotherapy $(y / n)$ & $3 / 7$ & $4 / 6$ & 1.00 \\
\hline CEA at diagnosis [mean $\pm \mathrm{SD}(\mathrm{ng} / \mathrm{mL})$ ] & $15.2 \pm 12.5$ & $7.7 \pm 7.7$ & 0.12 \\
\hline \multicolumn{4}{|l|}{ Colorectal tumor } \\
\hline Right colon/ left colon/ rectum & $3 / 3 / 4$ & $2 / 2 / 6$ & 0.67 \\
\hline \multicolumn{4}{|l|}{ Colorectal liver metastases } \\
\hline Number of lesions [median (IQR)] & $1(1-2)$ & $1(1-2)$ & 0.68 \\
\hline Larger diameter [mean \pm SD $(\mathrm{cm})]$ & $2.9 \pm 1.5$ & $2.0 \pm 1.2$ & 0.17 \\
\hline Laterality (unilateral /bilateral) & $9 / 1$ & $9 / 1$ & 1.00 \\
\hline Proximity of major vessel [(hilar or hepatic confluence) $(y / n)]$ & $0 / 10$ & $0 / 10$ & 1.00 \\
\hline Location (anterolateral / posterosuperior) & $8 / 2$ & $9 / 1$ & 1.00 \\
\hline
\end{tabular}

$\mathrm{ASA}=$ American Society of Anesthesiologist physical status score; $\mathrm{BMI}=$ body mass index; $\mathrm{CEA}=$ carcinoembryonic antigen; $\mathrm{SD}=$ standard deviation

treated by traditional open surgery (OPEN). Study inclusion criteria and patient flow are shown in Figure 1. The diagnosis of primary colorectal cancer was confirmed preoperatively by histology in all patients of both groups. The diagnosis of SCLM was confirmed postoperatively by histology in both groups as well.

The two groups were well matched and comparable in terms of preoperative clinical characteristics of patients, primary colorectal tumor and SCLM. Results are shown in Table 1.

\section{Intraoperative results}

The patients from LAP and OPEN group were comparable in terms of intraoperative variables. Results are detailed in Table 2.

Since extent of liver resection was one of the terms of propensity score matching, only minor liver resections were performed in both groups. A portal triad clamping (Pringle's maneuver) was not routinely used and was applied selectively, only in the case of bleeding with no significant difference in both groups. All patients with rectal cancer had either stoma covering low anastomosis or terminal colostomy.

\section{Postoperative short term outcomes: oncological results, clinical risk score, patient's recovery, morbidity and mortality}

There were no differences between LAP and OPEN group in terms of oncological results and CRS. All patients were found to have a CRS within the range of 1-4; no patients had a CRS 0 or 5 (Table 3). Results of patient's recovery, morbidity and mortality are detailed in Table 4 . No postoperative mortality occurred in either group. Fully description of morbidity and hospitalization of studied population are shown in Table 5.

\section{Postoperative long term outcome: recurrence and survival}

Of the 10 patients from OPEN group submitted to potentially curative simultaneous resection of primary colorectal cancer and SCLM four developed recurrent disease and, of these, all of them underwent repeat hepatic resection. Of the 10 patients from LAP two patients developed recurrence, which was both hepatic and extrahepatic and repeat resection was not feasible. Patterns and time 
TABLE 2. Intraoperative - simultaneous liver and colorectal surgery related variables

\begin{tabular}{|c|c|c|c|}
\hline Variable & $\begin{array}{c}\text { Simultaneous } \\
\text { Open } \\
(n=10)\end{array}$ & $\begin{array}{l}\text { Simultaneous } \\
\text { Laparoscopy } \\
(n=10)\end{array}$ & $\mathbf{P}$ \\
\hline Totally pure laparoscopic & - & 10 & - \\
\hline Conversion to laparotomy & - & 0 & - \\
\hline \multicolumn{4}{|l|}{ Liver surgery } \\
\hline Minor/major resection & $10 / 0$ & $10 / 0$ & 1.00 \\
\hline Atypical/segmentectomy/LLS & $6 / 2 / 2$ & $5 / 3 / 2$ & 0.87 \\
\hline Pringle's maneuver $(\mathrm{y} / \mathrm{n})$ & $2 / 8$ & $3 / 7$ & 1.00 \\
\hline \multicolumn{4}{|l|}{ Colorectal surgery } \\
\hline Colon/rectal resection & $6 / 4$ & $5 / 5$ & 1.00 \\
\hline Temporary stoma covering low anastomosis $(\mathrm{y} / \mathrm{n})$ & $2 * / 8$ & $3 * / 7$ & 1.00 \\
\hline Terminal colostomy (Hartman or APE) & $2 / 8$ & $2 / 8$ & 1.00 \\
\hline Operative time [mean \pm SD $(\mathrm{min})]$ & $257 \pm 66.8$ & $261 \pm 92.8$ & 0.91 \\
\hline \multicolumn{4}{|l|}{ Blood loss } \\
\hline Estimated [median (IQR) (mL)] & $170(70-230)$ & $105(30-180)$ & 0.23 \\
\hline Hemoglobin drop** $[$ median $(I Q R)(g / L)]$ & $22.5(9-28)$ & $15.5(9-17)$ & 0.38 \\
\hline Transfusion required $(y / n)$ & $3 / 7$ & $3 / 7$ & 1.00 \\
\hline
\end{tabular}

APE = abdominoperineal excision; LLS = left lateral sectionectomy; $*$ in both groups one patient required ileostomy after anastomotic leak; $* *$ Hemoglobin drop $=$ Hemoglobin preoperatively - Hemoglobin postoperatively $(\mathrm{g} / \mathrm{L})$

of recurrences, redo surgical procedures and longterm outcomes are shown in Table 5.

The median follow-up for surviving patients in the OPEN and LAP was 78 (61-130) and 24 (1-63) months, respectively $(\mathrm{P}=0.001)$. Among patients in OPEN OS/DFS was $100 \% / 90 \%$ at 1 year, $90 \% / 60 \%$ at 3 years, and $80 \% / 60 \%$ at 5 years. Among patients

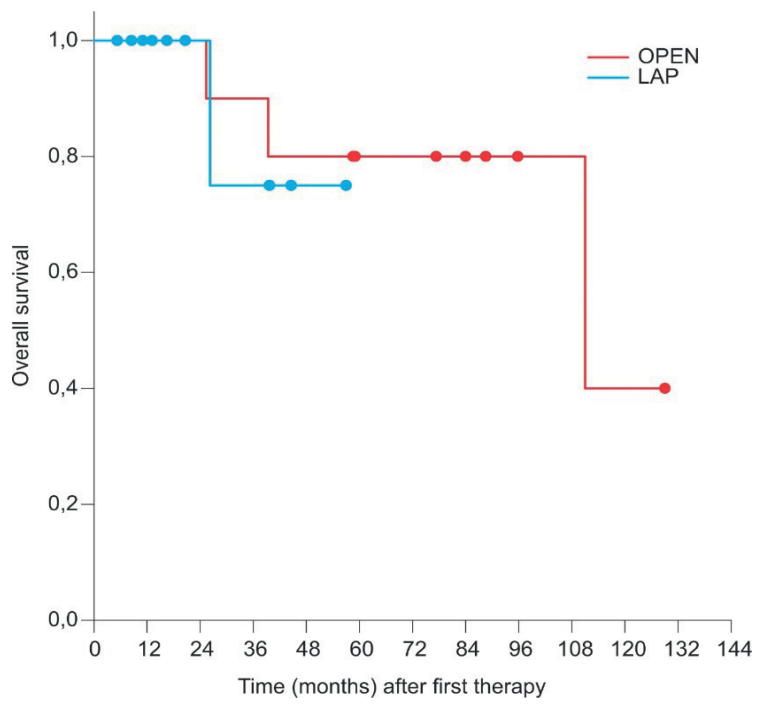

FIGURE 2. Kaplan-Meier estimates of overall survival between the simultaneous laparoscopy (blue line) and open surgery groups (red line). in LAP OS/DFS was $100 \% / 100 \%$ at 1 year, and $75 \% / 57 \%$ at 3 years. Simultaneous laparoscopic resection of the primary tumor and associated SCLM was first performed in this center in May 2012, thus the median follow-up period was too short to calculate the 5-year survival expectations for LAP group. There were no statistically significant dif-

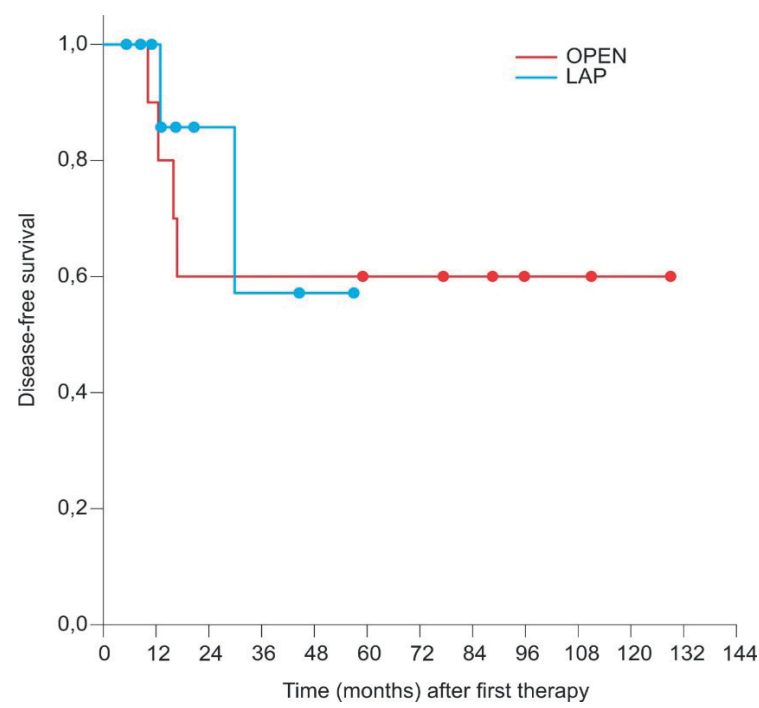

FIGURE 3. Kaplan-Meier estimates of disease-free survival between the simultaneous laparoscopy (blue line) and open surgery groups (red line). 
TABLE 3. Postoperative oncological results after patohistology and clinical risk score

\begin{tabular}{|c|c|c|c|}
\hline Variable & $\begin{array}{c}\text { Simultaneous } \\
\text { Open } \\
(n=10)\end{array}$ & $\begin{array}{l}\text { Simultaneous } \\
\text { Laparoscopy } \\
(\mathrm{n}=10)\end{array}$ & $\mathbf{P}$ \\
\hline \multicolumn{4}{|l|}{ Colorectal tumor } \\
\hline $\mathrm{T}(\mathrm{T} 1 / \mathrm{T} 2 / \mathrm{T} 3 / \mathrm{T} 4)$ & $0 / 1 / 8 / 1$ & $0 / 1 / 9 / 0$ & 1.00 \\
\hline $\mathrm{N}(\mathrm{NO} / \mathrm{N}+)$ & $2 / 8$ & $2 / 8$ & 1.00 \\
\hline Number of harvested lymph nodes (mean \pm SD) & $9.5 \pm 6.2$ & $13.7 \pm 6.9$ & 0.17 \\
\hline Well/moderately/poorly differentiated & $4 / 5 / 1$ & $4 / 6 / 0$ & 1.00 \\
\hline \multicolumn{4}{|l|}{ Negative surgical margin } \\
\hline Liver (y/n) & $10 / 0$ & $10 / 0$ & 1.00 \\
\hline Colorectal (y/n) & $10 / 0$ & $10 / 0$ & 1.00 \\
\hline Liver resection margin [median (IQR) $(\mathrm{mm})]$ & $2.5(2-5)$ & $5.0(1.8-8)$ & 0.38 \\
\hline $\operatorname{CRS}(1 / 2 / 3 / 4)$ & $2 / 5 / 2 / 1$ & $2 / 4 / 3 / 1$ & 0.96 \\
\hline
\end{tabular}

CRS = clinical risk score (no patients with CRS 0 or 5 in any of the group)

ferences between OPEN and LAP either in OS (P $=0.842)$ and DFS $(\mathrm{P}=0.724)$, respectively. KaplanMeier estimates of OS and DFS between the LAP and OPEN group are shown in Figures 2 and 3.

\section{Discussion}

The optimal strategy for resectable SCLM has not been established yet. In selected cases, the simultaneous surgery approach gives the advantages to avoid two surgical procedures thus reducing risk for patients and provides for economic savings while keeping acceptable morbidity and adequate oncologic results..$^{6-8}$ However, open resection of both primary tumor and SCLM often requires an extensive incision, especially if the location of the liver metastasis is opposite to the primary tumor location. Using laparoscopic approach exposure can be improved thus avoiding extensive laparotomies. ${ }^{20}$ Consequently, surgical stress and pain associated with large incisions can be reduced and patient's recovery enhanced. There is an ongoing effort to show the potential benefit of totally laparoscopic strategies for the radical treatment of stage IV colorectal cancer. ${ }^{12-30,35}$

The present study was designed to investigate the perioperative results, oncologic outcomes and survival of patients undergoing pure laparoscopic simultaneous resection of both the primary colorectal cancer and SCLM. During this study period, the choice of intervention was subject to selection bias since simultaneous laparoscopic liver procedures were reserved for the most ideal candidates. Moreover, the laparoscopic treatment of patients with an extraperitoneal rectal cancer remains clinically challenging compared to upper rectal or colon cancer. To minimize these biases, the LAP group was compared to OPEN group by propensity score matching on the factors known to influence the choice of the approach. Importantly, there were no differences in patient demographics, tumor characteristics, or the extent of the operation, so the present characteristics well identify the most ideal candidates to a combined laparoscopic liver and colorectal surgery. Patients selected for this approach were without severe comorbidities (ASA I-II in majority of cases), with a solitary (median number: 1), small (median diameter: 2 $\mathrm{cm}$ ), unilateral SCLM, located in accessible anterolateral segments and were mostly resectable by atypical wedge resections or left lateral sectionectomy. Consequently, only minor liver resections were performed. These results are consistent with previous studies showing well selected patients and demonstrating a high rate of minor $(89 \%)$ and nonanatomical resections $(60 \%))^{17-27,35}$ In this study colorectal cancer characteristics did not preclude the possibility to perform a laparoscopic surgery as well with majority of patients presenting a T3 primary tumor. There were no differences in $\mathrm{N}$ stage and importantly, the number of harvested lymph nodes was even higher in the LAP group. Half of the patients in the LAP group presented with rectal cancer. Surprisingly, previous studies have demonstrated that despite the high rate of rectal resections in some series, the number of the temporary ileostomies was low. $25,27,35$ The results of the present study contradict this: all of the rectal cancer patients had either temporary ileostomy 
TABLE 4. Postoperative outcome: patient's recovery, morbidity and mortality. No postoperative mortality occurred in either group. Adjuvant chemotherapy and incisional hernias

\begin{tabular}{|c|c|c|c|}
\hline Variable & $\begin{array}{c}\text { Simultaneous } \\
\text { Open } \\
(n=10)\end{array}$ & $\begin{array}{l}\text { Simultaneous } \\
\text { Laparoscopy } \\
(n=10)\end{array}$ & $\mathbf{P}$ \\
\hline ICU stay & 0 & 0 & - \\
\hline HDU stay [median (IQR) (days)] & $4(2-6)$ & $3(2-5)$ & 0.59 \\
\hline Solid food oral intake [median (IQR) (days)] & $5.5(4-6)$ & $3(3-4)$ & 0.006 \\
\hline Stool passing [median (IQR) (days)] & $4.5(4-5)$ & $4(3-5)$ & 0.46 \\
\hline Intravenous narcotics requirement [median (IQR) (days)] & $6.5(6-7)$ & $4.5(3-7)$ & 0.08 \\
\hline Hospital stay [median (IQR) (days)] & $11.5(10-33)$ & $8(8-12)$ & 0.044 \\
\hline \multicolumn{4}{|l|}{ Morbidity } \\
\hline Overall (y/n) & $5 / 5$ & $3 / 7$ & 0.65 \\
\hline Liver specific* $(y / n)$ & $2 / 8$ & $0 / 10$ & 0.47 \\
\hline$C D<I I I(y / n)$ & $2 / 8$ & $2 / 8$ & 1.00 \\
\hline CD Illab (y/n) & $3 / 7$ & $1 / 9$ & 0.58 \\
\hline$C D>I I I(y / n)$ & $0 / 10$ & $0 / 10$ & 1.00 \\
\hline Mortality $(y / n)$ & $0 / 10$ & $0 / 10$ & 1.00 \\
\hline Postoperative chemotherapy $(y / n)$ & $5 / 5$ & $2 / 8$ & 0.35 \\
\hline Incisional hernias (y/n) & $3 / 7$ & $0 / 10$ & 0.21 \\
\hline
\end{tabular}

HDU = high dependency unit; ICU = intensive care unit; *Liver specific complications included liver failure, bile leak, bile collection or liver hemorrhage; values in bold are significant at $\mathrm{P}<0.05$

covering low anastomosis or terminal colostomy. This finding might have been due to a high rate of preoperative radiotherapy, which highlights that most rectal resections were performed for cancer in the lower rectum. Moreover, according to some reports simultaneous resection generally is considered unsuitable for rectal cancer due to a high rate of anastomotic leakage. ${ }^{36}$ Another possible explanation is a concern regarding prolonged vascular clamping which is responsible for transient portal hypertension with edema of the intestinal mucosa that might be leading to colorectal anastomotic failure. ${ }^{21,35}$

The feasibility of simultaneous laparoscopic operations was clearly demonstrated in the present study: no conversion to open surgery has been performed. This result reflects a findings of a recent review, where a conversion rate of only $0.7 \%$ has been reported. ${ }^{35}$ Similarly, Tranchart et al. reported a low conversion rate of $7 \%$ in their large multicenter study. ${ }^{30}$ It has been highlighted, that simultaneous resections executed by two different specialized teams allowing good results in terms of conversion rates and perioperative outcomes. ${ }^{35}$ Of note, the present study was performed at high-volume tertiary referral center, where all procedures were performed by an expert surgical team, composed of experienced colorectal and hepatobiliary surgeons. Controversy still exist, which procedure should be carried out first and many authors reported colorectal resection was the first step of the simultaneous surgery. ${ }^{15-16,20,22,29-30}$ Instead, in the present study liver resection precede colorectal surgery and the same strategy has been reported by others. ${ }^{18-19,21,35}$ The underlying rationality is that the resection of liver metastases requires a low central venous pressure to minimize the blood loss and the preceding liver resection will not interfere with the subsequent fluid resuscitation in the process of colorectal resection. In addition, the choice of carrying out the liver resection as first step of treatment gives to the surgeon the opportunity to change surgical strategy from a simultaneous procedure to a "liver first" resection which has been showed to be another effective treatment of SCLM. ${ }^{5}$ Moreover, liver metastases are the main determinant of patient prognosis and the present authors believe, that SCLM are leading the decision making process: if simultaneous strategies are not feasible, then the metastases should be managed first. However, no decisions were modified and all procedures were finished simultaneously.

In terms of intraoperative measurements of outcome, the median operating time of $261 \mathrm{~min}$ after LAP is comparable with the time reported by Jung et al..$^{25}$, and is shorter than the time reported by 
TABLE 5. Morbidity details, hospital stay, patterns and time of recurrence, redo procedures and long-term outcome

\begin{tabular}{|c|c|c|c|c|c|c|}
\hline Patients & Morbidity & $\begin{array}{c}\text { Hospital } \\
\text { stay (days) }\end{array}$ & Recurrence location (years after first surgery) & Redo & $\begin{array}{l}\text { Survival } \\
\text { (years) }\end{array}$ & $\begin{array}{l}\text { Survival status } \\
\text { (March 2017) }\end{array}$ \\
\hline 1 & $\begin{array}{l}\text { Wound infection } \\
\text { (CD II) }\end{array}$ & 12 & $\begin{array}{l}\text { 1) Liver (2Y) } \\
\text { 2) Liver (3Y) }\end{array}$ & $\begin{array}{l}\text { 1) Liver } \\
\text { 2) / }\end{array}$ & 3 & DOD \\
\hline 2 & Bile collection (CD Illa) & 36 & Liver (1Y) & Liver & 7 & NED \\
\hline 3 & nil & 9 & $\begin{array}{c}\text { 1) Liver (1Y) } \\
\text { 2) Colon (3Y) } \\
\text { 3) Peritoneal carcinosis (4Y) }\end{array}$ & $\begin{array}{l}\text { 1) Liver } \\
\text { 2) Colon } \\
\text { 3) / }\end{array}$ & 4 & DOD \\
\hline 5 & nil & 10 & no & / & 11 & NED \\
\hline 6 & Pneumonia (CD ॥) & 12 & no & I & 10 & D - other \\
\hline 7 & nil & 11 & no & l & 8 & NED \\
\hline 8 & nil & 9 & no & l & 8 & NED \\
\hline 1 & nil & 8 & no & / & 5 & NED \\
\hline 2 & Anastomotic leak (CD IIIb) & 42 & Liver and peritoneal carcinosis ( $1 Y$ ) & / & 2 & DOD \\
\hline 3 & nil & 7 & no & l & 4 & NED \\
\hline 4 & nil & 7 & Liver and peritoneal carcinosis (3Y) & l & 4 & AWD \\
\hline 5 & nil & 8 & no & / & 2 & NED \\
\hline 6 & nil & 12 & no & / & 2 & NED \\
\hline 7 & nil & 8 & no & l & 1 & NED \\
\hline 8 & $\begin{array}{l}\text { Pulmonary embolism } \\
\text { (CD II) }\end{array}$ & 9 & no & l & 1 & NED \\
\hline
\end{tabular}

$\mathrm{AWD}=$ alive with disease; $C D=$ Clavien Dindo classification of complication severity; $\mathrm{D}$ - other = death without recurrence of disease; $\mathrm{DOD}=\mathrm{dead}$ of disease; $\mathrm{LAP}=$ simultaneous $\mathrm{AWD}=$ alive with disease; $\mathrm{CD}=\mathrm{Clavien}$ Dindo classification of complication severity; $\mathrm{D}-$ other = dea
laparoscopic surgery; $\mathrm{OPEN}=$ simultaneous open surgery; NED = no evidence of disease; $Y=$ years

others, where it was more than 300 min. ${ }^{13-16,19,22-24,30}$ Nevertheless, operative time was not different between OPEN and LAP group. This result is inconsistent with some previous studies showing that the duration of operation was significantly longer in the laparoscopic groups. ${ }^{15,22,25}$ A possible explanation can be, that in contrast to present study, these reports included major liver resections as well, which are known to prolong operative time. However, several authors reported equivalent operative times for both groups. ${ }^{16,23,30}$

Intraoperative blood loss is a major concern especially in hepatic resection and perioperative transfusion has been associated with a poor prognosis. ${ }^{37}$ The findings of decreased blood loss with the laparoscopic approach have been reported in several studies, explained in part by the laparoscopic magnification, and decreased venous oozing from the cut surface under pneumoperito- neum. ${ }^{15-16,22-23}$ Results of present study contradicts this as laparoscopic approach did not allow diminishing blood loss and transfusion rate. Contrast to prior studies, in this analysis blood loss was not based only on estimation but it has been objectivized by measuring the volume of blood in the suction canister and precisely weighing the absorbed blood in the sponges. Moreover, the pre- and postoperative hemoglobin levels were demonstrated exactly as well. The reason for this was to exclude the possibility of differences in preoperative hemoglobin levels which can contribute to the difference in blood loss and transfusion rate between two groups since it is well known that patients with SCLM can develop anemia. Moreover, it has been suggested that the blood loss can be underestimated especially in the open surgery. ${ }^{38}$ Despite analyzing additional objective variable in this study, there were no differences between groups. It should be 
noted that outcome observed after open surgery was excellent as well (median estimated blood loss $170 \mathrm{ml}$ ). Similarly, several recent studies have suggested that there is no decreased blood loss and transfusion rate in the laparoscopic group. ${ }^{19,25,30}$

In respect to postoperative oncological results, a high CRS has been found to be an independent negative prognostic predictor in previous studies. 32,39 The impacts of CRS and colorectal tumor differentiation on survival analysis were eliminated, since the OPEN and LAP groups were comparable in that terms as well. The risk of inadequate oncologic resection is the major concern for the use of laparoscopic resection for malignancy. A positive surgical margin has been shown to predict worse DFS after resection. ${ }^{40}$ Castaing et al. emphasized that the increase in $\mathrm{R} 1$ resections did not affect OS and suggest it might reflect complex anatomic locations of metastases adjacent to major vascular structures. ${ }^{41}$ To avoid an inherent selection bias with more difficult cases potentially being relegated to open approach, it is important to point out that the two groups of the present study were perfectly matched in term of SCLM proximity to major vessels (hilar or hepatic confluence) as well; there were no tumors with such characteristics in any of groups. Importantly, complete $\mathrm{R} 0$ resections of both the primary and SCLM were achieved in all patients confirming the reports of others, which reported a high rate of $\mathrm{R} 0$ resections of $100 \%$ and $90 \%$ as well. ${ }^{25,30}$ Surprisingly, the rate of R0 resections has been not reported by many authors. . $^{14-16,22}$ The width of the surgical margin was not different between two groups and interestingly, the width was even larger in the LAP group. Moreover, a recent study failed to demonstrate that the width of negative margin correlated with recurrence or survival..$^{42}$ Importantly, in this study neither peritoneal carcinomatosis nor port site metastases after simultaneous resections of SCLM by laparoscopic means were found. These results suggest that laparoscopic procedure does not compromise oncologic principles.

The major findings in this study were lesser length of hospital stay and faster solid food oral intake for LAP group. The median hospital stay of 8 days in the LAP group is similar to or shorter than the time reported by others, where it has been in a range from 7.4 to 16 days. The benefit of decreased hospital stay was recognized in these analyses as well. ${ }^{15-30}$ Interestingly, substantial geographical differences exist which depends on national health care systems. Takasu et al. confirmed the benefit of lesser length of hospital stay which were 16 days for the laparoscopy and 36 days for the open group..$^{23}$ However, Tranchart et al. found no differences in the length of stay between laparoscopic and open groups, and noted that simultaneous resections still appear as difficult procedures for surgeons, which could have influence their decisions concerning patient's hospital discharge. ${ }^{30}$ Patients undergoing laparoscopic procedure resumed solid food oral intake earlier, however it has no impact on earlier stool passing in this study, thus the benefit of this parameter remains questionable. Similarly, several authors reported a shorter time to resume a bowel movement and starting an oral intake. ${ }^{19,25}$

In terms of other postoperative measurements of outcome none of the patients required intensive care unit stay and the high dependency unit stay was comparable between groups. Ferretti et al. reported a median one-day intensive care unit stay. ${ }^{29}$ However, these relevant parameters were not reported in several previous analyses. ${ }^{15-28,35}$

Pain control is one of the potential advantage of laparoscopy and in this study, it was evaluated by intravenous narcotics requirement. Laparoscopy offered a benefit of less narcotic requirements, patients in the LAP group needed intravenous narcotics for 4.5 days compared with the OPEN group where these were necessary for 6.5 days. Notwithstanding, statistical significance was reached only marginally $(\mathrm{P}=0.08)$. Hu et al. reported that patients having undergone laparoscopic resection had less severe postoperative pain, but it is not clear how they asses this parameter. ${ }^{19}$ Surprisingly, in several other reports on this topic pain control was not investigated. ${ }^{13-18,20-30}$ Some surgeons combined a laparoscopic colorectal resection with an open procedure for the liver, as reported by Hatwell et al..$^{20}$ However, this technique also left a large incision in the upper abdomen, and the advantage of laparoscopy was not realized fully.

The present study found that LAP and OPEN were not significantly different in terms of postoperative morbidity and mortality. Of note, no liver specific morbidity was observed in the LAP group. Nevertheless, some bile collections which occurred in the OPEN group were easily managed by percutaneous drainage. Importantly, there were no organ failures and postoperative deaths in two groups either. Similarly, no differences in morbidity and zero mortality were reported in several other analyses. ${ }^{15-16,19,22-23}$ Polignano et al. reported that the surgical morbidity was mainly related to the colonic surgery. ${ }^{18}$ Contrary, in the present series only one anastomotic leak occurred in OPEN and one in the LAP group, respectively. Jung et al. reported a 
higher rate of minor complications in the open surgery group (superficial surgical site infections and adhesive ileus), which seem to be strongly related to the presence of the bigger wound. ${ }^{25}$ However, the number of minor complications were equal in the present analysis. Interestingly, despite laparoscopy has the potential to reduce the incisional hernia rates, none of the studies on the present topic investigated this parameter which is responsible for long-term morbidity. ${ }^{12-30,35}$ In the present study none of the LAP whereas three patients from the OPEN group developed incisional hernia, but the difference was not significant.

In the present study, neither the 3-year OS nor the DFS was found to be different for LAP compared with the OPEN group. Similarly, no differences were found between groups in terms of OS reported by others .15-16,19,22-23,30 After long and adequate median follow-up of 78 months this analysis revealed excellent long-term results among patients in OPEN with 5-year OS/DFS of 80 and 60\%, respectively. However, due to a shorter follow-up period, only mid-term results were available for the LAP group with 3-year OS of 75\% and DFS of 57\%, respectively. Similarly, in the current literature some of the authors reported the short- and midterm results only and the 3-year OS rates ranged from 52 to $78 \%{ }^{15,30}$ Unfortunately, data regarding recurrence were inconsistently reported in several case-matched series on this topic. ${ }^{15-16,19,23,25}$

The present study is a subject to a number of limitations. First and foremost, only a small cohort of patients from a single center were analyzed which were collected across a long interval of time. However, the majority of case-matched studies in the literature derived from a small single center series, which included from 7 to 24 patients. ${ }^{15-16,19,22-23,25}$ Even in the largest multicenter analysis, which included 142 patients from 14 centers the average number of patients per hospital was ten. ${ }^{29}$ It demonstrates clearly the limited indications of simultaneous laparoscopic procedures even in the most experienced centers worldwide. Second, given the retrospective nature of this study, selection bias may have been present. Although propensity score matching was performed to overcome potential bias and to make the two groups similar it is less effective than a prospective randomized trial. Notwithstanding, to conduct a randomized study on this topic is still an unresolved issue. Third, since laparoscopic simultaneous procedures started in 2012 the median follow-up data between groups differs and the excellent long-term survival results achieved with OPEN are still waiting to be proved by LAP. However, except for introduction of laparoscopy, the management of SCLM was homogeneous over the study period and the same surgical team made the treatment decisions and performed the procedures.

Indeed, the power of the present study is limited, but importantly the two surgical approaches brought about similar outcomes in terms of postoperative morbidity, mortality, survival and recurrence. Nevertheless, some surgeons may insist on traditional open approach and not to accept widespread changes in clinical practice given the lack of several investigated variables to support the superiority of laparoscopic approach. However, the results of a recently published studies investigating inflammatory markers are promising and have a potential to prove that the reduced inflammatory response by laparoscopy might have positive impact on oncogenesis. ${ }^{43}$

\section{Conclusions}

Based on limited evidence, LAP patients experienced some clinically relevant perioperative benefits without compromise of oncologic outcomes. In high-volume centers experienced in both laparoscopic colorectal and liver surgery, simultaneous minimally invasive approach is appropriate in well selected patients presenting with limited SCLM.

\section{References}

1. Torre LA, Bray F, Siegel RL, Ferlay J, Lortet-Tieulent J, Jemal A. Global cancer statistics, 2012. CA Cancer J Clin 2015; 65: 87-108. doi: 10.3322/caac.21262

2. Leporrier J, Maurel J, Chiche L, Bara S, Segol P, Launoy G. A population-based study of the incidence, management and prognosis of hepatic metastases from colorectal cancer. Br J Surg 2006; 93: 465-74. doi: 10.1002/bjs.5278

3. Adams RB, Aloia TA, Loyer E, Pawlik TM, Taouli B, Vauthey JN. Selection for hepatic resection of colorectal liver metastases: expert consensus statement. HPB 2013; 15: 91-103. doi: 10.1111/j.1477-2574.2012.00557.x

4. Yoshidome H, Kimura F, Shimizu H, Ohtsuka M, Kato A, Yoshitomi H, et al Interval period tumor progression: does delayed hepatectomy detect occult metastases in synchronous colorectal liver metastases? J Gastrointest Surg 2008; 12: 1391-8. doi: 10.1007/s11605-008-0540-9

5. Mentha G, Majno PE, Andres A, Rubbia-Brandt L, Morel P, Roth AD. Neoadjuvant chemotherapy and resection of advanced synchronous liver metastases before treatment of the colorectal primary. Br J Surg 2006; 93: 872-8. doi: $10.1002 /$ bjs.5346

6. Fahy BN, Fischer CP. Synchronous resection of colorectal primary and hepatic metastasis. J Gastrointest Oncol 2012; 3: 48-58. doi: 10.3978/j. issn.2078-6891.2012.004

7. Ejaz A, Semenov E, Spolverato G, Kim Y, Tanner D, Hundt J, et al. Synchronous primary colorectal and liver metastasis: impact of operative approach on clinical outcomes and hospital charges. HPB 2014; 16: 1117-26. doi: 10.1111/hpb.12302 
8. Muangkaew P, Cho JY, Han HS, Yoon YS, Choi Y, Jang JY, et al. Outcomes of simultaneous major liver resection and colorectal surgery for colorectal liver metastases. J Gastrointest Surg 2016; 20: 554-63. doi: 10.1007/s11605015-2979-9

9. Lacy AM, Delgado S, Castells A, Prins HA, Arroyo V, Ibarzabal A, et al. The long-term results of a randomized clinical trial of laparoscopy-assisted versus open surgery for colon cancer. Ann Surg 2008; 248: 1-7. doi: 10.1097/ SLA.0b013e31816a9d65

10. Park JS, Choi GS, Jun SH, Park SY, Kim HJ. Long-term outcomes after laparoscopic surgery versus open surgery for rectal cancer: a propensity score analysis. Ann Surg Oncol 2013; 20: 2633-40. doi: 10.1245/s10434013-2981-y

11. Wakabayashi G, Cherqui D, Geller DA, Buell JF, Kaneko H, Han HS, et al. Recommendations for laparoscopic liver resection: a report from the second international consensus conference held in Morioka. Ann Surg 2015; 261: 619-29. doi: 10.1097/SLA.0000000000001184

12. Leung KL, Lee JF, Yiu RY, Ng SS, Li JC. Simultaneous laparoscopic resection of rectal cancer and liver metastasis. J Laparoendosc Adv Surg Tech A 2006; 16: 486-8. doi: 10.1089/lap.2006.16.486

13. Sasaki A, Nitta H, Otsuka K, Takahara T, Nishizuka S, Wakabayashi G. Ten-year experience of totally laparoscopic liver resection in a single institution. $\mathrm{Br} \mathrm{J}$ Surg 2009; 96: 274-9. doi: 10.1002/bjs.6472

14. Tranchart H, Diop PS, Lainas P, Pourcher G, Catherine L, Franco D, et al. Laparoscopic major hepatectomy can be safely performed with colorectal surgery for synchronous colorectal liver metastasis. HPB 2011; 13: 46-50. doi: 10.1111/j.1477-2574.2010.00238.x

15. Huh JW, Koh YS, Kim HR, Cho CK, Kim YJ. Comparison of laparoscopic and open colorectal resections for patients undergoing simultaneous RO resection for liver metastases. Surg Endosc 2011; 25: 193-8. doi: 10.1007/ s00464-010-1158-z

16. Chen KY, Xiang GA, Wang HN, Xiao FL. Simultaneous laparoscopic excision for rectal carcinoma and synchronous hepatic metastasis. Chin Med J 2011; 124: $2990-2$.

17. Hoekstra LT, Busch OR, Bemelman WA, van Gulik TM, Tanis PJ. Initial experiences of simultaneous laparoscopic resection of colorectal cancer and liver metastases. HPB Surg 2012; 893956. doi: 10.1155/2012/893956

18. Polignano FM, Quyn AJ, Sanjay P, Henderson NA, Tait IS. Totally laparoscopic strategies for the management of colorectal cancer with synchronous liver metastasis. Surg Endosc 2012; 26: 2571-8. doi: 10.1007/s00464-012-2235-2

19. Hu MG, Ou-yang CG, Zhao GD, Xu DB, Liu R. Outcomes of open versus laparoscopic procedure for synchronous radical resection of liver metastatic colorectal cancer: a comparative study. Surg Laparosc Endosc Percutan Tech 2012; 22: 364-9. doi: 10.1097/SLE.0b013e31825af6b2

20. Hatwell C, Bretagnol F, Farges O, Belghiti J, Panis Y. Laparoscopic resection of colorectal cancer facilitates simultaneous surgery of synchronous liver metastases. Colorectal Dis 2013; 15: e21-8. doi: 10.1111/codi.12068

21. Spampinato MG, Mandalá L, Quarta G, Del Medico P, Baldazzi G. One-stage, totally laparoscopic major hepatectomy and colectomy for colorectal neoplasm with synchronous liver metastasis: safety, feasibility and short-term outcome. Surgery 2013; 153: 861-5. doi: 10.1016/j.surg.2012.06.007

22. Lin $Q$, Ye $Q$, Zhu $D$, Wei $Y$, Ren $L$, Zheng $P$, et al. Comparison of minimally invasive and open colorectal resections for patients undergoing simultaneous RO resection for liver metastases: a propensity score analysis. Int J Colorectal Dis 2015; 30: 385-95. doi: 10.1007/s00384-014-2089-2

23. Takasu C, Shimada M, Sato H, Miyatani T, Imura S, Morine Y, et al. Benefits of simultaneous laparoscopic resection of primary colorectal cancer and liver metastases. Asian J Endosc Surg 2014; 7: 31-7. doi: 10.1111/ases.12066

24. Ando K, Oki E, Ikeda T, Saeki H, Ida S, Kimura Y, et al. Simultaneous resection of colorectal cancer and liver metastases in the right lobe using pure laparoscopic surgery. Surg Today 2014; 44: 1588-92. doi: 10.1007/s00595013-0801-4

25. Jung KU, Kim HC, Cho YB, Kwon $\mathrm{CH}$, Yun SH, Heo JS, et al. Outcomes of simultaneous laparoscopic colorectal and hepatic resection for patients with colorectal cancers: a comparative study. J Laparoendosc Adv Surg Tech A 2014; 24: 229-35. doi: 10.1089/lap.2013.0475

26. Ida S, Oki E, Ando K, Kimura Y, Yamashita Y, Saeki H, et al. Pure laparoscopic right-sided hepatectomy in the semi-prone position for synchronous colorectal cancer with liver metastases. Asian J Endosc Surg 2014; 7: 133-7. doi: 10.1111/ases.12098
27. Berti S, Francone E, Minuto M, Bonfante P, Sagnelli C, Bianchi $C$, et al. Synchronous totally laparoscopic management of colorectal cancer and resectable liver metastases: a single center experience. Langenbecks Arch Surg 2015; 400: 495-503. doi: 10.1007/s00423-015-1281-3

28. Takorov I, Belev N, Lukanova T, Atanasov B, Dzharov G, Djurkov V, et al. Laparoscopic combined colorectal and liver resections for primary colorectal cancer with synchronous liver metastases. Ann Hepatobiliary Pancreat Surg 2016; 20: 167-72. doi: 10.14701/ahbps.2016.20.4.167

29. Ferretti S, Tranchart H, Buell JF, Eretta C, Patriti A, Spampinato MG, et al. Laparoscopic simultaneous resection of colorectal primary tumor and liver metastases: results of a multicentric international study. World J Surg 2015; 39: 2052-60. doi: 10.1007/s00268-015-3034-4

30. Tranchart H, Fuks D, Vigano L, Ferretti S, Paye F, Wakabayashi G, et al. Laparoscopic simultaneous resection of colorectal primary tumor and liver metastases: a propensity score matching analysis. Surg Endosc 2016; 30 : 1853-62. doi: 10.1007/s00464-015-4467-4

31. Strasberg SM, Belghiti J, Clavien PA, Gadzijev E, Garden JO, Lau WY, et al. The Brisbane 2000 Terminology of Liver Anatomy and Resections. HPB 2000; 2: 333-9.

32. Fong $\mathrm{Y}$, Fortner J, Sun RL, Brennan MF, Blumgart LH. Clinical score for predicting recurrence after hepatic resection for metastatic colorectal cancer: analysis of 1001 consecutive cases. Ann Surg 1999; 230: 309-18; discussion 318-21.

33. Dindo D, Demartines N, Clavien PA. Classification of surgical complications: a new proposal with evaluation in a cohort of 6336 patients and results of a survey. Ann Surg 2004; 240: 205-13.

34. Austin PC. A comparison of 12 algorithms for matching on the propensity score. Stat Med 2014; 33: 1057-69. doi: 10.1002/sim.6004

35. Garritano S, Selvaggi F, Spampinato MG. Simultaneous minimally invasive treatment of colorectal neoplasm with synchronous liver metastasis. Biomed Res Int 2016; 9328250. doi: 10.1155/2016/9328250

36. Peeters KC, Tollenaar RA, Marijnen CA, Klein Kranenbarg E, Steup WH, Wiggers $\mathrm{T}$, et al. Risk factors for anastomotic failure after total mesorectal excision of rectal cancer. Br J Surg 2005; 92: 211-6. doi: 10.1002/bjs.4806

37. Kooby DA, Stockman J, Ben-Porat L, Gonen M, Jarnagin WR, Dematteo RP, et sl. Influence of transfusions on perioperative and long-term outcome in patients following hepatic resection for colorectal metastases. Ann Surg 2003, 237: 860-9; discussion 869-70. doi: 10.1097/01.SLA.0000072371.95588.DA

38. Schiffman SC, Kim KH, Tsung A, Marsh JW, Geller DA. Laparoscopic versus open liver resection for metastatic colorectal cancer: a metaanalysis of 610 patients. Surgery 2015; 157: 211-22. doi: 10.1016/j.surg.2014.08.036

39. Ivanecz A, Kavalar R, Palfy M, Pivec V, Sremec M, Horvat M, et al. Can we improve the clinical risk score? The prognostic value of $\mathrm{p} 53, \mathrm{Ki}-67$ and thymidylate synthase in patients undergoing radical resection of colorectal liver metastases. HPB 2014; 16: 235-42. doi: 10.1111/hpb.12089

40. Pawlik TM, Scoggins CR, Zorzi D, Abdalla EK, Andres A, Eng C, et al. Effect of surgical margin status on survival and site of recurrence after hepatic resection for colorectal metastases. Ann Surg 2005; 241: 715-22; discussion 722-4.

41. Castaing D, Vibert E, Ricca L, Azoulay D, Adam R, Gayet B. Oncologic results of laparoscopic versus open hepatectomy for colorectal liver metastases in two specialized centers. Ann Surg 2009; 250: 849-55. doi: 10.1097/ SLA.0b013e3181bcaf63

42. Postriganova N, Kazaryan AM, Røsok BI, Fretland ÅA, Barkhatov L, Edwin B. Margin status after laparoscopic resection of colorectal liver metastases: does a narrow resection margin have an influence on survival and local recurrence? HPB 2014; 16: 822-9. doi: 10.1111/hpb.12204

43. Fretland AA, Sokolov A, Postriganova N, Kazaryan AM, Pischke SE, Nilsson $\mathrm{PH}$, et al. Inflammatory response after laparoscopic versus open resection of colorectal liver metastases. Medicine (Baltimore) 2015; 94: e1786. doi: 10.1097/MD.0000000000001786 